\title{
Cyclopentane hydrate-based processes for treating heavy metal containing wastewater
}

\author{
Yamei Yang, Hang Zhou, Feng Li, Changrui Shi, Shuai Wang, and Zheng Ling* \\ Key Laboratory of Ocean Energy Utilization and Energy Conservation of Ministry of Education, School of Energy \& Power Engineering, \\ Dalian University of Technology, 116024 Dalian, China
}

\begin{abstract}
The scarcity of water and increasing water pollution are the pressing challenge human being facing. Recovering water and valuable heavy metals is highly desired for treating heavy metal containing wastewater. We proposed a novel hydrate-based process to treat $\mathrm{Ni}^{2+}$ containing wastewater. The water recovery, $\mathrm{Ni}^{2+}$ enrichment factor, desalination efficiency were studied using this cyclopentane hydrate-based method. A water recovery of $43 \%$ can be obtained with a desalination efficiency of round $88 \%$ and an enrichment factor of 1.6. The desalination efficiency and the quality of the as-made water via the hydratebased process can be further improved to above $99 \%$ via three-stage hydrate reaction. The proposed hydrate-based water treatment process may find wide applications in waste water treatment and heavy metal recycling.
\end{abstract}

\section{Introduction}

The freshwater crisis is getting worse due to the dramatic increase in water consumption and severe water contamination.[1] The discharge of wastewaters, particularly the ones containing heavy metal ions, to environment without treatment results in a big problem, both in terms of environment.

Nickel, as one of the heavy metal, is a common contaminant which exists in the wastewater from electroplating, battery manufacture, painting, etc..[2] Nickel can cause great health risks when exposes to human bodies. Therefore, it is highly desired to reduce the amount of nickel containing wastewater and properly treat the outlet water before discharge. So a large number of processes have been developed to treat the heavy metal containing wastewater, such as ion exchange, adsorption, chemical precipitation, membrane filtration, and so on.[2, 3] All these methods have their advantages, however, they all focus on the heavy metals. Usually, the contents of heavy metals are low and variable. Taking nickel for example, the nickel concentration in the discharged wastewater is extremely variable, depending on source. For example, the content of nickel is 2-900 $\mathrm{mg} / \mathrm{L}$ for the electroplating outlet water and $0-40 \mathrm{mg} / \mathrm{L}$ for the one from painting manufacture.[4] So it usually takes plenty of time and large numbers of chemical agents to treat the wastewater.

It would be ideal to develop novel methods to extract water from wastewater and enrich the heavy metals. Hydrate-based processes have been confirmed to be promising for this purpose. Hydrates are solid crystals, being constituted by water molecules (also called host molecules) and small molecules (also named as guest molecules).[5] Hydrates will be formed when water and guest molecules contact at proper condition, such as low temperature and high pressure. The formed solid hydrate can be easily separated from salty water and decomposes into fresh water and recyclable guest molecules. The water content of hydrate is $85 \mathrm{~mol} \%$, so it means that using a few guest molecule can extract large amount of water. Additionally, the guest molecules can be recycled and the formation can be conducted above the ice point at atmospheric pressure, making this method attractive.

Although hydrate-based processes have been widely used for seawater desalination,[6] there are few reports on their application for treating heavy metal containing wastewater.[7, 8]

In this study, we proposed a hydrate-base process to treat $\mathrm{Ni}^{2+}$ containing wastewater. The impact of reation time, water recovery, $\mathrm{Ni}^{2+}$ enrichment factor, desalintion efficiency and mutli-stage treatment were studied. The results confirmed that the hydrate-based process is very promising for treating heavy metal containing wastewater, with high water revocery, high desalination efficiency and short treatment time.

\section{Experimental section}

\subsection{Materials}

The chemical agents with analytical pure were bought from Sinopharm Chemical Company, and used without further purification. Graphite powders (2000 mesh) were bought from Shanghai Macklin Biochemical Co., Ltd. The deionized water was made in our lab using reverse osmosis, having a specific resistance of $18 \mathrm{M} \Omega . \mathrm{NiCl}_{2}$

\footnotetext{
* Corresponding author: zling@dlut.edu.cn
} 
aqueous solutions with various concentrations were used as the mimic wastewater.

\subsection{Experimental procedure}

In a typic run, $\mathrm{NiCl}_{2}$ aqueous solution was mixed with cyclopentane at a volume ratio of $3: 1$. Graphite was added with a dosage of $50 \mathrm{mg}$ per $80 \mathrm{~mL}$ mixtute. Hydrate formation was conducted at $2{ }^{\circ} \mathrm{C}$ under constantly magnetic stirring at a speed of $600 \mathrm{rpm}$. All the hydration foramtion reactions run for $12 \mathrm{~h}$. The Raman analysis was conducted using $532 \mathrm{~nm}$ laser (LabRAM HR Evolution, Horiba)

After the reactions, hydrate solids were separated via vacuum filtration and subjected centrifugation at $-5^{\circ} \mathrm{C}$ and $6000 \mathrm{rpm}$ for 3 minutes to fully remove the trapped brine in hydates. The separated hydrates were decompoesed at room temperature, producing desalted water and cyclopentane. The volume of the as-produced water was meansured. The conductivity of as-produced water was measured using conductivity meter (ET915, eDAQ TECH, Australia). The concentration of the asmade water and original $\mathrm{NiCl}_{2}$ aqueous solutions were calculated using a calibration curve based on the conductivity curve (see Figure 1).

The performance of hydrate-based process via multistage for treating $\mathrm{Ni}^{2+}$ containing wastewater was studied. The $\mathrm{Ni}^{2+}$ concentration of the feeding solution is 1000 $\mathrm{mg} / \mathrm{L}$, the produced water was used as the feeding solution for the following stage. (1),

The water recovery was calculated using Equation

$$
\text { Water recovery }=100 \% * V_{f} / V_{0}
$$

where $V_{0}$ is the volume of the feeding solution, $V_{f}$ is the as-produced water by decomposition of hydrate.

Desalination efficiency was calculated using Equation (2),

$$
\text { Desalination efficiency }=\left(C_{0}-C_{f}\right) * 100 \% / C_{0}
$$

where $C_{0}$ is the original concentration of the feeding solution, $C_{f}$ is the concentration of the as-produced water by decomposition of hydrate.

The $\mathrm{Ni}^{2+}$ enrichment factor was calculated using Equation (3),

$$
\mathrm{Ni}^{2+} \text { enrichment factor }=100 \% * C_{r} / C_{0}
$$

Where $C_{0}$ is the original concentration of the feeding solution, $C_{r}$ is the concentration of the residual solution after separation of the as-produced hydrate.

\section{Results and discussion}

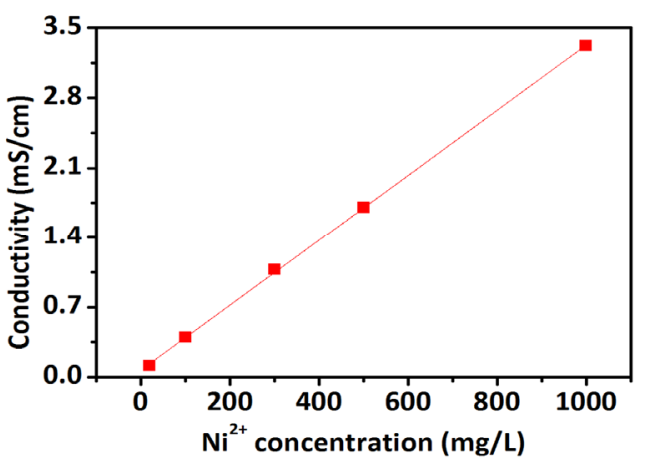

Fig. 1. The linear relation between $\mathrm{Ni}^{2+}$ concentration and the conductivity of its aqueous solution. The solutions were made by dissolving various amount of $\mathrm{NiCl}_{2}$ in deionized water.

The content of heavy metal ions is relative low in most contaminated wastewater. It is well-know that at low concentration the concentration of salt solution and its conductivity has a linear relation. As shown in Figure 1, the conductivity of $\mathrm{NiCl}_{2}$ aqueous solution with $\mathrm{Ni}^{2+}$ concentration ranging from 20 to $1000 \mathrm{mg} / \mathrm{L}$ was tested. The concentration and conductivity shows good linear relationship with a $\mathrm{R}^{2}$ equating 0.9998 . So we use the tested conductivity to calculate the concentration of produced water and residual solution in this study.

As shown in Figure $2 \mathrm{a}, \mathrm{NiCl}_{2}$ aqueous solution and cyclopentane are immiscible, graphite powders are locating at the interface of the $\mathrm{NiCl}_{2}$ aqueous solution and cyclopentane. After reaction of $12 \mathrm{~h}$, the mixture turned into slurry with low flowability (see Figure 2b) due to the formation of large amount of solid cyclopentane hydrate. The Raman spectrum, as shown in Figure 2c, confirms the produced solid is cyclopentane hydrate. The peak at $895 \mathrm{~cm}^{-1}$ is corresponding to the ring breathing of cyclopentane.[9] The peaks locating at 2874 and $2981 \mathrm{~cm}^{-1}$ are corresponding to the $\mathrm{C}-\mathrm{H}$ stretching modes of symmetric and asymmetric vibrations of cyclopentane, respectively.[10]

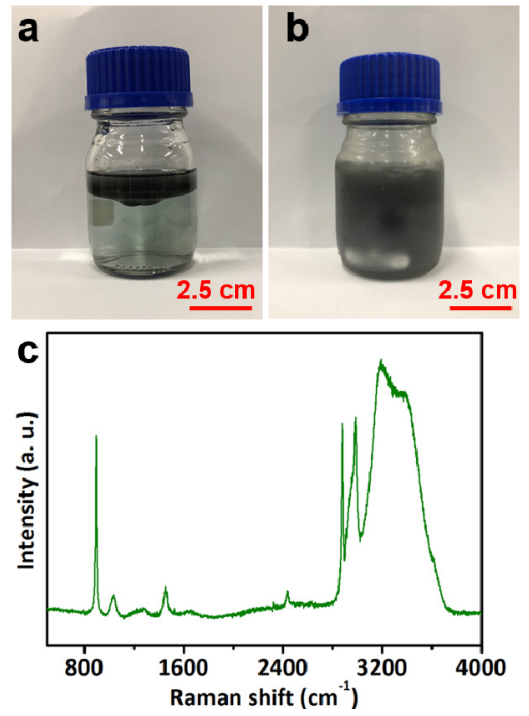

Fig. 2. (a) Digital images of mixture of $\mathrm{NiCl}_{2}$ aqueous solution and cyclopentane with graphite powders and mixture after hydrate formation, (b) Raman spectrum of the formed hydrate solid. 
It is well known that salts are effective thermodynamic inhibitors for both ice and hydrates formation. Their presence will inevitably reduce the freezing point and require large degree of supercooling. However, cyclopentane hydrate can form at $2^{\circ} \mathrm{C}$ which is above the freezing point of ice, confirming the great promising in energy saving of this hydrate-based method.[11]

The mechanism for hydrate-based desalination is that during the process of hydrate formation, water molecules from the heavy metal contained solution will form cages via hydrogen bonds, encaging cyclopentane. The solid hydrates will be separated from residual solution and decompose into fresh water and recyclable cyclopentane. The salts were not encaged into the cages, so theoretically pure water will be produced during the hydrate-based process. Since cyclopentane locates inside the cage are made of water molecules, increasing the stability of the cages, hydrates can be formed above the freezing point of ice.

The water recovery is an important parameter to indicate the performance of the method for water treatment. It can be seen in Figure 3 that our method has a water recovery of around $43 \%$ (the average) when the feeding solution has a low concentration. This large water recovery is comparable or superior to the mainstream processes for water treatment, such as reverse osmosis.[6] The water recovery decreases as the concentration of the feeding solution increases, while around $30 \%$ water can still be extracted from the mimic solution with a $\mathrm{Ni}^{2+}$ concentration as high as $1000 \mathrm{mg} / \mathrm{L}$. It is easy to understand that the activity of water will be reduced as the salt content increases. With the decreased water activity, the ability of water to form hydrogen bonded cages will be impaired. It is worth to mention that the impairing is limited as the water recovery only dropped by $30 \%$ as the concentration increased by a factor of 50 . The relatively stable water recovery indicates this method is feasible for treating waste water with a wide concentration range.

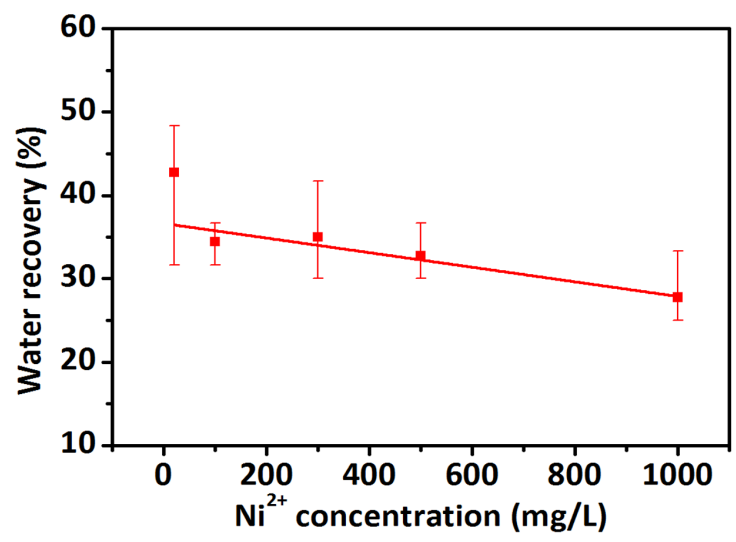

Fig. 3. Effect of $\mathrm{Ni}^{2+}$ concentration on the water recovery from mimic wastewater via formed cyclopentane hydrates. All the experiments were conducted with graphite as a promoter with a formation period of 12 hours.

When the water in the $\mathrm{Ni}^{2+}$ containing solution was extracted via hydrate, the residual solution will be concentrated. As shown in Figure 4, we studied the concentration-dependent $\mathrm{Ni}^{2+}$ enrichment factor. It can be seen that the enrichment factor ranges from 1.15 to 1.60. In consonance with the water recovery, the $\mathrm{Ni}^{2+}$ enrichment factor also decreases with the increased concentration in the feeding solution. As mentioned earlier, water activity will decrease with the increased $\mathrm{Ni}^{2+}$ concentration, so it is getting harder to extract water from the feeding solution and concentrate the solution.

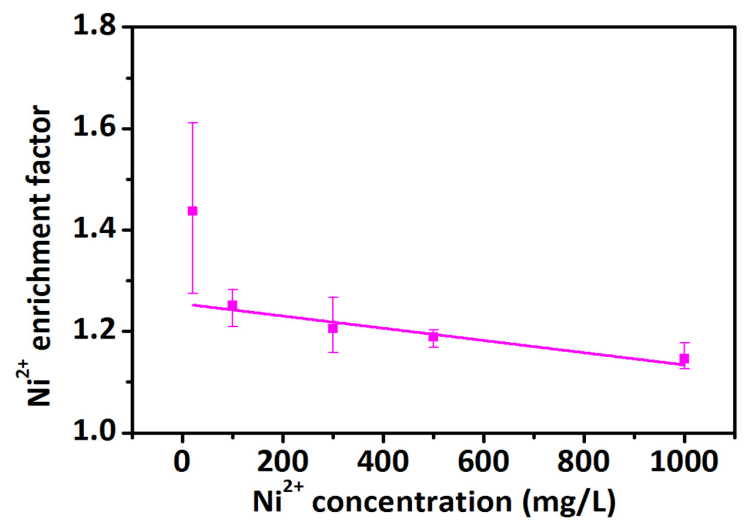

Fig. 4. Effect of $\mathrm{Ni}^{2+}$ concentration on $\mathrm{Ni}^{2+}$ enrichment ratio via formed cyclopentane hydrates. All the experiments were conducted using graphite as a promoter with a formation period of 12 hours.

The desalination efficiency determines the quality of the as-produced water. So we measured the conductivity of the as-produced water via the hydrate based process. It turns out that the desalination efficiency ranges from $62 \%$ to $88 \%$, which is quite common for the hydratebased process.[11, 12] It was reported that the trapped salt by solid hydrate is responsible for the adequate desalination efficiency. With proper post treatment, the desalination efficiency can be further improved to above $95 \%$.[13] It is worth to note that the desalination efficiency is improved as the $\mathrm{Ni}^{2+}$ concentration increasing. It is similar with ice that there is pre-melting at the surface. The pre-melting will produce water to wash away the residual salt. Additionally, the decomposition of as-made hydrate will be promoted by high $\mathrm{Ni}^{2+}$ concentration salts, producing more water to wash away the residual salt. The centrifugation will remove the washing liquid, resulting in the improved desalination efficiency.

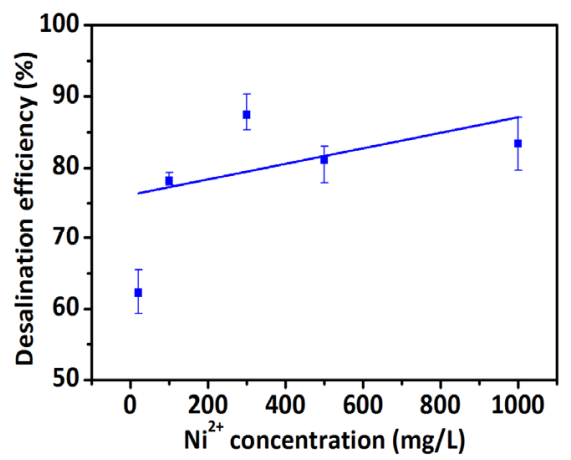

Fig. 5. Effect of $\mathrm{Ni}^{2+}$ concentration on the desalination efficiency via formed cyclopentane hydrates. All the 
experiments were conducted using graphite as a promoter with a formation period of 12 hours.

In order to further improve the quality of the asproduced water, we conducted multi-stage desalination via the hydrate-based processes using a feeding solution with $\mathrm{Ni}^{2+}$ concentration of $1000 \mathrm{mg} / \mathrm{L}$. As shown in Figure 6, the one-stage desalination has an efficiency of $84 \%$, while the efficiency can be improved to $96 \%$ by a two-stage treatment. An efficiency of $99.2 \%$ can be obtained via the three-stage treatment, indicating this hydrate-based process can produce water with high quality.

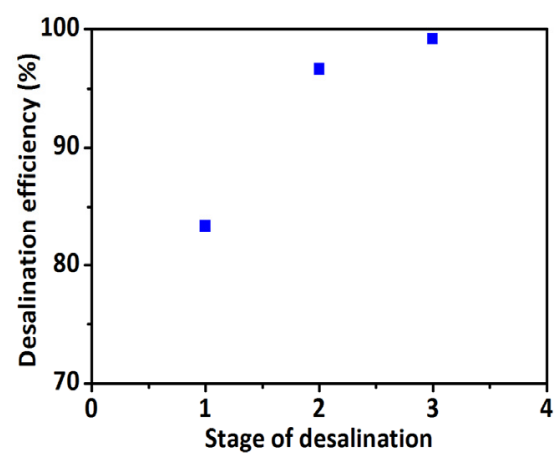

Fig. 6. The performance of hydrate-based process via multistage for treating $\mathrm{Ni}^{2+}$ containing wastewater

\section{Conclusions}

This study shows that hydrate-based process has great promising in treating $\mathrm{Ni}^{2+}$ containing waste water. Cyclopentane hydrate can be formed at $2^{\circ} \mathrm{C}$ in $\mathrm{NiCl}_{2}$ solution with a $\mathrm{Ni}^{2+}$ concentration ranging from $20-1000$ $\mathrm{mg} / \mathrm{L}$. The water recovery can be as large as $43 \%$ with a desalination efficiency range of $62-88 \%$. The efficiency and the quality of the as-produced water can be further improved to above $99 \%$ via multi-stage hydrate reaction. The proposed hydrate-based water treatment process may find wide applications in seawater desalination, waste water treatment and heavy metal recycling.

\section{Acknowledgement}

This research was financially supported by the National Natural Science Foundation of China (No. 51606027) and the Fundamental Research Funds for the Central Universities of China (DUT17RC(4)23).

\section{References}

1. C.J. Vörösmarty, P.B. McIntyre, M.O. Gessner, D. Dudgeon, A. Prusevich, P. Green, S. Glidden, S.E. Bunn, C.A. Sullivan, C.R. Liermann, P.M. Davies, Global threats to human water security and river biodiversity, Nature, 467 (2010) 555.

2. L. Gürel, Applications of the Biosorption Process for Nickel Removal from Aqueous Solutions - A Review, Chemical Engineering Communications, 204 (2017) 711-722.
3. Ihsanullah, A. Abbas, A.M. Al-Amer, T. Laoui, M.J. Al-Marri, M.S. Nasser, M. Khraisheh, M.A. Atieh, Heavy metal removal from aqueous solution by advanced carbon nanotubes: Critical review of adsorption applications, Separation and Purification Technology, 157 (2016) 141-161.

4. Y.F. Lam, L.Y. Lee, S.J. Chua, S.S. Lim, S. Gan, Insights into the equilibrium, kinetic and thermodynamics of nickel removal by environmental friendly Lansium domesticum peel biosorbent, Ecotoxicology and Environmental Safety, 127 (2016) 61-70.

5. E.D. Sloan Jr, Fundamental principles and applications of natural gas hydrates, Nature, 426 (2003) 353.

6. P. Babu, A. Nambiar, T. He, I.A. Karimi, J.D. Lee, P. Englezos, P. Linga, A Review of Clathrate Hydrate Based Desalination To Strengthen EnergyWater Nexus, ACS Sustainable Chemistry \& Engineering, 6 (2018) 8093-8107.

7. H. Dong, L. Zhang, Z. Ling, J. Zhao, Y. Song, The controlling factors and ion exclusion mechanism of hydrate-based pollutant removal, ACS Sustainable Chemistry \& Engineering, 7 (2019) 7932-7940.

8. Y. Song, H. Dong, L. Yang, M. Yang, Y. Li, Z. Ling, J. Zhao, Hydrate-based heavy metal separation from aqueous solution, Scientific Reports, 6 (2016) 21389.

9. Q. Lv, L. Li, X. Li, Z. Chen, Formation Kinetics of Cyclopentane + Methane Hydrates in Brine Water Systems and Raman Spectroscopic Analysis, Energy \& Fuels, 29 (2015) 6104-6110.

10. C. Lo, J. Zhang, P. Somasundaran, J.W. Lee, Raman Spectroscopic Studies of Surfactant Effect on the Water Structure around Hydrate Guest Molecules, The Journal of Physical Chemistry Letters, 1 (2010) 2676-2679.

11. F. Li, Z. Chen, H. Dong, C. Shi, B. Wang, L. Yang, Z. Ling, Promotion effect of graphite on cyclopentane hydrate based desalination, Desalination, 445 (2018) 197-203.

12. Y.-N. Lv, S.-S. Wang, C.-Y. Sun, J. Gong, G.-J. Chen, Desalination by forming hydrate from brine in cyclopentane dispersion system, Desalination, 413 (2017) 217-222.

13. S. Han, J.-Y. Shin, Y.-W. Rhee, S.-P. Kang, Enhanced efficiency of salt removal from brine for cyclopentane hydrates by washing, centrifuging, and sweating, Desalination, 354 (2014) 17-22. 